\title{
Notes
}

Selçuk Özyurt*

\section{Building Reputation in a War of Attrition Game: Hawkish or Dovish Stance?}

DOI 10.1515/bejte-2015-0093

Published online May 25, 2016

Abstract: This paper examines a two-player war of attrition game in continuoustime, where (1) fighting (i. e., escalating the conflict) is costless for a player unless he quits, (2) at any point in time, each player can attack to his opponent and finalize the game with a costly war, (3) there is two-sided uncertainty regarding the players' resolve, and (4) each player can choose his tone/stance (either hawkish or dovish) at the beginning of the game, which affects his quitting cost. The results imply that choosing hawkish (dovish) regime is optimal if and only if the benefit-cost ratio of the dispute is sufficiently high (low). If hawkish tone is going to give a player upper hand in a dispute, then choosing a more aggressive tone does not increase his payoff. However, choosing a more dovish tone increases a player's payoff whenever dovish regime is optimal.

Keywords: war of attrition game, continuous time games, reputation, dispute resolution

JEL Classification: C72, D74, D82, D83

In politics... never retreat, never retract... never admit a mistake.

Napoleon Bonaparte

\section{Introduction}

The current paper generalizes the results of Ozyurt (2014) and extends its workhorse war of attrition model by adding an initial stage where the players can endogenously choose their escalation cost parameters. What is novel about the game is that (1) escalating the conflict (i. e., fighting) is costless for a player provided that he never retreats, (2) at any point in time, each player can choose one of three possible

*Corresponding author: Selçuk Özyurt, Department of Economics, Harvard University, 1805 Cambridge St. Cambridge, MA 02138, USA; Faculty of Arts and Social Sciences, Sabancı University, Orhanli Tuzla 34956, Istanbul, Turkey, E-mail: ozyurt@sabanciuniv.edu 
actions; escalate (wait), back down (quit), and attack, (3) there is two-sided uncertainty regarding the players' commitment (i. e., resolve), and finally (4) each player can choose his stance, tone, or regime (either hawkish or dovish) at the beginning of the game, which affects the cost he will suffer when he quits. Sending strong public threats to the opponent, pledging himself to a certain course of action or to some bold, future act can be interpreted as choosing hawkish tone in a dispute because these actions raise public's expectation, and so cause bigger embarrassment in case of a failure of meeting these expectations. ${ }^{1}$

An international dispute between two states and a labor dispute between a firm and a union are two examples that would fit to the setup provided above. Mimicking the resolved type by escalating the dispute is potentially a costly action for a rational player because quitting after escalating the dispute for a while is costly. Attacking is also a costly action. However, both of these actions have signaling values, and so, they may provide a leverage to a player during the war of attrition game. A player can build his reputation by mimicking the resolved type. Higher reputation for resolve intimidates the rival and forces him to play a (mixed) strategy in which he quits with a greater probability. Clearly, a player's ex ante payoff increases as his opponent quits with a greater probability. On the other hand, the threat of a costly war introduces some sort of deadline effect on reputation building. A rational player does not quit beyond time, at which his escalation costs exceed his war cost, and so, low war cost or greater sensitivity to escalation costs implies a shorter deadline. By escalating the conflict, a rational player signals that he is committed to attack (with a positive probability) at some time after his deadline if the game ever reaches to this time. Therefore, if a player's deadline is shorter and if he escalates the dispute, then his rival will have to build his reputation faster, which means that his rival will play a strategy such that he quits with a greater probability.

By choosing his regime, a player can control how fast he can build his reputation on his resolve and how short his deadline for attacking will be. Dovish stance gives the ability of building reputation at a faster rate. ${ }^{2}$ Hawkish regime, on the other hand, shortens the players' deadline for attacking. Therefore, a rational player will either choose the dovish regime and build his

1 Leaders make use of this mechanism infrequently (Snyder and Borghard 2011; Trachtenberg 2012). For example, the United Kingdom made very mild threats to Iran during the Abadan Crisis of 1951 (dovish stance) but issued serious warnings to Argentina during the Falkland Islands crisis in 1982.

2 Note that given his strategy, a player's reputation at time $t$ is the conditional probability that this player is the resolved type, conditional on the event that the player has not yet quit prior to time $t$. Choosing the dovish stance yields smaller growth rate for the cost of quitting. Thus, a player who chooses the dovish stance can optimally play a strategy in which he quits at a greater rate, and so builds his reputation faster. 
reputation faster or the hawkish regime and commit to a shorter deadline. Our results show that choosing the hawkish regime (and so committing to a shorter deadline) is optimal when the benefit-cost ratio of the dispute is sufficiently high. Otherwise, choosing the dovish regime (i. e., building reputation at a faster rate) is optimal. These results were speculated in Ozyurt (2014), but formally proved in the current paper. In addition, the current results show that having more hawkish tone never benefits a player if hawkish regime is already the optimal one. However, choosing more dovish tone increases a player's payoff whenever the dovish regime is optimal. Furthermore, hawkish tone may be advantageous in a dispute only if the players have the option of finishing the dispute with a costly war, such as starting a combat in international disputes or strike in labor disputes.

War of attrition game is first proposed by Maynard Smith (1974) and is useful for the study of a wide variety of conflict situations. ${ }^{3}$ This paper is primarily related to the reputation and bargaining literature initiated by Myerson (1991). Myerson investigates the impacts of one-sided reputation building on bilateral negotiations. Abreu and Gul (2000), Kambe (1999), Compte and Jehiel (2002), Atakan and Ekmekci (2014), and Ozyurt (2014, 2015) consider twosided versions of it. The structure of the war of attrition game in this paper is similar to the one studied in Fearon (1994). However, there are two fundamental differences between the two: Fearon assumes that (1) players are known to be flexible, but there is some uncertainty regarding the players' war costs, and (2) the players' escalation costs are exogenous.

Ozyurt (2014) is the most related work to the current paper. It challenges conventional wisdom - in international relations literature - that the ability of generating higher escalation (i. e., audience) costs is an advantage for a leader of a state. In particular, Ozyurt (2014) shows that lower escalation costs may give a player upper hand in a crisis depending on (1) the benefit-cost ratio of the dispute, (2) initial probability of resolve, and (3) how fast states generate escalation costs with time. The current paper takes a step further, generalizes the results of Ozyurt (2014) for a larger class of escalation cost functions, and extends its workhorse model by adding an initial stage where the players can

3 Examples in the literature range from patent races (Fudenberg et al. 1983) to bargaining (Ordover and Rubinstein 1986; Osborne 1985; Chatterjee and Samuelson 1987) to public good provision (Bliss and Nalebuff 1984), and price wars and exit in oligopolistic markets (Ghemawat and Nalebuff 1985; Fudenberg and Tirole 1986). Hendricks, Weiss, and Wilson (1988) present a very general analysis of the war of attrition game with complete information. Bishop, Cannings, and Maynard Smith (1978), Riley (1980), Milgrom and Weber (1985), Nalebuff and Riley (1985), Ponsati and Sakovics (1995), and Bulow and Klemperer (1999) present analysis of the war of attrition game with incomplete information. 
endogenously choose their escalation cost parameters. This extension allows the possibility of studying not only the optimal type (hawkish vs. dovish) but also the optimal intensity of regime choice in a dispute.

Section 2 explains the details of the three-stage, infinite-horizon, continuous-time war of attrition game. Section 3 characterizes the third-stage equilibrium strategies. Section 4 presents the main results: I study four particular examples and examine how players' regime choices change with the characteristics of the dispute. Finally, Section 5 concludes.

\section{The War of Attrition Game}

\subsection{Timing}

Two players, 1 and 2, are in dispute over a prize worth $v_{i}>0$ for each player $i \in\{1,2\}$. The dispute (war of attrition game) is a three-stage infinite-horizon, continuous-time game. All stages start and the first two stages end at time 0 . In stage 1, players simultaneously choose and announce their messages, regimes, or tones $m_{i} \in\{h, d\}:=M$ for $i=1,2$. Each player can choose either the hawkish $(h)$ or the dovish $(d)$ regime.

A player knows that he will never be forced to commit to a hardline policy, but is uncertain about his opponent. Therefore, each player believes that nature sends one of two messages $\{r, f\}$ to his opponent in stage 2. A player who receives the message $r$, resolved, is constrained to follow a hardline policy; he never quits and never attacks. If a player receives the message $f$, flexible, then he will continue to play the game with no commitment. The players share the same belief that player $i$ receives the message $r$ with probability $z_{i}$ where $z_{i} \in(0,1)$. Therefore, the probability of being resolved is independent of the chosen messages.

Upon the beginning of the third stage (still at time 0 ), the players begin to play a war of attrition game, where at all times $t \geq 0$ before the dispute ends, each player can choose to escalate (wait), quit (back down), or attack. The dispute ends when one or both players attack or quit.

\subsection{Payoffs}

If either player attacks before the other quits, then the dispute ends with "war" and each player $i$ receives the (net expected) payoff $-w_{i}<0$. It indicates all the 
risks and gains resulting from war. The case $-w_{i}<0$ indicates that staying away from the dispute is more desirable for a player than attacking once the player is involved in it. If player $i$ picks message $m_{i} \in M$ and quits at time $t \geq 0$ before the other quits or attacks, then its opponent $j$ receives the prize while player $i$ suffers escalation costs equal to $c_{i}^{m_{i}}(t)$, a continuous and strictly increasing function of the amount of escalation. I assume that $0 \leq c_{i}^{m_{i}}(t), c_{i}^{m_{i}}(0)<v_{i}$, and $c_{i}^{d}(t) \leq c_{i}^{h}(t)$ for all $i$ and $t$. The last condition implies that the hawkish stance leads to a (weakly) higher escalation costs.

If one player chooses to attack at time $t$ and the other chooses to quit or attack at the same time, then both flexible players receive their war payoffs $-w_{i}$. However, if both quit at time $t$, then flexible player $i$ receives $\frac{v_{i}}{2}-c_{i}^{m_{i}}(t)$ given that $i$ 's regime choice was $m_{i}$ in the first stage. These particular assumptions are not crucial because in equilibrium, simultaneous concessions or attacks occur with probability 0. Finally, if players escalate the conflict indefinitely, each flexible player gets a payoff that is strictly less than his war payoff. ${ }^{4}$ Call this war of attrition game where all the parameters are common knowledge $G$.

\subsection{Strategies}

The only source of uncertainty is the players' actual types, which matters only in the third stage of the game. In the first stage, all players are flexible (rational) in the sense that they choose their strategies, given their beliefs, to maximize their expected payoffs. At the beginning of the game each player knows that his future self will be flexible. However, each player is uncertain about his opponent's future self: following the second stage, the opponent is either flexible or resolved.

The strategy of the resolved type is simple: never quit and never attack during the war of attrition game. Resolved types' payoffs are ignored because they are redundant for the analysis. What motivates and interests the subsequent analyses are the optimal (i. e., equilibrium) strategies of the flexible types. Equilibrium strategies of the third stage of the game are in mixed strategies, and so they are intricate objects. For pedagogical reasons, I first present what pure

4 Flexible players' payoff at time infinity would be interpreted as payoff of "perpetual conflict avoidance." By neglecting to address high-conflict situations, avoiders risk allowing problems to fester out of their control. By assuming that flexible players' payoffs of perpetual conflict avoidance are worse than their war payoff, we ensure that the flexible players are confrontational. That is, the flexible players prefer confrontation even if the conflict may end badly for them. 
strategies may look like and the payoffs that they correspond to. Then I provide the general description for the strategies and payoffs.

A pure strategy for player $i$ in the third stage of the game $G$ is a tuple $\left(Q_{i}(\bar{m}), A_{i}(\bar{m})\right) \in \mathbb{R}_{+}^{2}$ for each $\left(m_{1}, m_{2}\right):=\bar{m} \in M$, where $Q_{i}(\bar{m})$ and $A_{i}(\bar{m})$ denote the times at which player $i$ quits and attacks, respectively. Therefore, the strategies of the resolved type of player $i$ are $Q_{i}(\bar{m})=A_{i}(\bar{m})=\infty$. Suppose that players choose $\left(m_{1}, m_{2}\right)=\bar{m} \in M$ in stage 1 and players $i$ and $j$, for $i, j=1,2$ and $j \neq i$, are flexible.

- If $A_{i}(\bar{m}) \leq \min \left\{A_{j}(\bar{m}), Q_{i}(\bar{m}), Q_{j}(\bar{m})\right\}$, then the payoffs to players $i$ and $j$ are $-w_{i}<0$ and $-w_{j}<0$, respectively.

- If $Q_{i}(\bar{m})<\min \left\{A_{i}(\bar{m}), A_{j}(\bar{m}), Q_{j}(\bar{m})\right\}$, then the payoffs to players $i$ and $j$ are $-c_{i}^{m_{i}}\left(Q_{i}(\bar{m})\right)<0$ and $v_{j}>0$, respectively.

- If $Q_{i}(\bar{m})=Q_{j}(\bar{m})<\min \left\{A_{i}(\bar{m}), A_{j}(\bar{m})\right\}$, then the payoffs to players $i$ and $j$ are $\frac{v_{i}}{2}-c_{i}^{m_{i}}\left(Q_{i}(\bar{m})\right)$ and $\frac{v_{j}}{2}-c_{j}^{m_{j}}\left(Q_{j}(\bar{m})\right)$, respectively.

- If $Q_{i}(\bar{m})=Q_{j}(\bar{m})=A_{i}(\bar{m})=A_{j}(\bar{m})=\infty$, then the payoffs to players $i$ and $j$ are some $-M \in \mathbb{R}_{-}$, where $\max \left\{w_{i}, w_{j}\right\}<M$.

More formally, a mixed strategy of flexible player $i$ in the first stage is a function $\mu_{i}: M \rightarrow[0,1]$, where $\mu_{i}(m)$ denotes the probability that flexible player $i$ chooses the message $m \in M$. Note that $\mu_{i}(h)+\mu_{i}(d)=1$ for $i=1,2$.

Player $i$ 's third stage (mixed) strategy, $\left\{Q_{i}^{\bar{m}}, A_{i}^{\bar{m}}\right\}_{\bar{m} \in M^{2}}$, has two parts. For each message profile $\bar{m}=\left(m_{1}, m_{2}\right) \in M^{2}$, a right-continuous distribution function $Q_{i}^{\bar{m}}(t): \mathbb{R}_{+} \cup\{\infty\} \rightarrow[0,1]$ represents the probability that player $i$ quits by time $t$ (inclusive). Similarly, for each message profile $\bar{m}$, a continuous distribution function $A_{i}^{\bar{m}}(t): \mathbb{R}_{+} \cup\{\infty\} \rightarrow[0,1]$ represents the probability that player $i$ attacks by time $t$ (inclusive). If player $i$ 's strategy, following the message profile $\bar{m}$, is $Q_{i}^{\bar{m}}(t)$, then flexible player i's strategy is $Q_{i}^{\bar{m}}(t) /\left(1-z_{i}\right)$ because the resolved type never quits. The same arguments hold for $A_{i}^{\bar{m}}(t)$. Note that $Q_{i}^{\bar{m}}(t)+A_{i}^{\bar{m}}(t) \leq 1$ for all $0 \leq t$ and $\lim _{t \rightarrow \infty} A_{i}^{\bar{m}}(t) \leq 1-z_{i}$ for all $i$ and $\bar{m}$.

Given a strategy profile $\sigma=\left\langle\mu_{i},\left\{Q_{i}^{\bar{m}}, A_{i}^{\bar{m}}\right\}_{\bar{m} \in M^{2}}\right\rangle_{i=1}^{2}$, in the subgame following the message realization $\bar{m}$, flexible player $i$ 's expected payoff of quitting at time $t$ is

$$
\begin{aligned}
U_{i}^{q}(t, \sigma, \bar{m})= & v_{i} Q_{j}^{\bar{m}}(t)-w_{i} A_{j}^{\bar{m}}(t)+\left[1-Q_{j}^{\bar{m}}(t)-A_{j}^{\bar{m}}(t)\right]\left[-c_{i}^{m_{i}}(t)\right] \\
& +\left(\frac{v_{i}}{2}-c_{i}^{m_{i}}(t)\right)\left[Q_{j}^{\bar{m}}(t)-Q_{j}^{\bar{m}}\left(t^{-}\right)\right],
\end{aligned}
$$

with $Q_{j}^{\bar{m}}\left(t^{-}\right)=\lim _{y \uparrow t} Q_{j}^{\bar{m}}(y)$. Similarly, flexible player i's expected payoff of attacking at time $t$ is

$$
U_{i}^{a}(t, \sigma, \bar{m})=v_{i} Q_{j}^{\bar{m}}(t)-w_{i}\left[1-Q_{j}^{\bar{m}}(t)\right] .
$$


Therefore, flexible player $i$ 's expected payoff in the subgame following the message realization $\bar{m}$ is

$$
U_{i}(\sigma, \bar{m})=\int_{t \in[0, \infty)} \frac{U_{i}^{q}(t, \sigma, \bar{m})}{1-z_{i}} d Q_{i}(t)+\int_{t \in[0, \infty)} \frac{U_{i}^{a}(t, \sigma, \bar{m})}{1-z_{i}} d A_{i}(t)
$$

Finally, flexible player i's expected payoff in the game is

$$
U_{i}(\sigma)=\sum_{\bar{m} \in M^{2}} \mu(\bar{m}) U_{i}(\sigma, \bar{m})
$$

where for all $\bar{m}=\left(m_{1}, m_{2}\right) \in M^{2}, \mu(\bar{m})=\mu_{1}\left(m_{1}\right) \mu_{2}\left(m_{2}\right)$.

\subsection{Discussion}

Escalation costs can be interpreted as the players' opportunity costs of quitting. That is, it is the total value of the opportunities that are missed or not used effectively by backing down from the hardline policy. Public commitments that players make during a dispute are expected to serve an agenda the players may form. This agenda may include items that are not directly related to the prize (e.g., increasing audiences' support and the likelihood of winning upcoming elections, building reputation for future negotiations, discouraging potential rivals, and preventing future disputes etc.). Therefore, a player's expected value of following a hardline policy may increase with time because as time passes, the player is more likely to convince his audiences about his resolve, and persuaded audiences would increase the likelihood of the successful execution of his agenda. As a result, if the expected benefit of following the hardline policy increases in time, then the opportunity cost of backing down is expected to increase in time as well. Alternatively, escalation costs may represent a player's psychological disutility - due to social disapproval or embarrassment - of quitting. ${ }^{5}$

A player may commit not to back down in a dispute for reasons other than the prospects of the dispute. For example, a leader may be resolved because of his firm belief that backing down, and thus giving up for the prize, is simply a

5 The increasing cost of escalation is a widely used concept in international relations literature, which is known as audience costs. This concept is first proposed by Fearon (1994). Audience costs may occur if the leader of a state makes public threats or promises but fails to carry through on them. In the international relations literature, there is a considerable amount of discussion concerning micro foundations of audience costs. For instance, a leader that backs down from a public commitment may suffer audience costs because the leader is less likely to be reelected (Smith 1998), has violated national honor (Fearon 1994), or has lost his reputation and the credibility of his rhetoric (Sartori 2002; Guisinger and Smith 2002). 
decision that will not be ratified by his supporters. Therefore, the positive priors (i. e., $z_{i}$ 's) can be interpreted as the players' initial beliefs on the existence of such motives that may force their opponents to be resolved. Resolved types closely resemble the commitment types that are first defined by Myerson (1991) ( $r$-insisting types) and studied further by Abreu and Gul (2000) and Kambe (1999). These strategy types are first used in games by Kreps and Wilson (1982) and Milgrom and Roberts (1982), where commitments are modeled as behavioral types that exist in society so that rational players can mimic them if it is optimal to do so. In a bilateral negotiation, commitment types always demand a particular share and accept an offer if and only if it weakly exceeds that share. ${ }^{6}$

\section{Equilibrium for the Third Stage of the War of Attrition Game}

Because most of the arguments in this section are direct generalization of the analysis of Ozyurt (2014), readers are advised to refer to this paper for a more detailed discussion. All the proofs are deferred to Appendix.

Lemma 1: If a strategy profile $\sigma=\left\langle\mu_{i},\left\{Q_{i}^{\bar{m}}, A_{i}^{\bar{m}}\right\}_{\bar{m} \in M^{2}}\right\rangle_{i=1}^{2}$ constitutes a sequential equilibrium of the game $G$, then for each $\bar{m}=\left(m_{1}, m_{2}\right) \in M^{2}$, there exists a finite number $t_{\bar{m}}^{\star}>0$ such that, for $i=1,2$, the following conditions hold:

1. $Q_{i}^{\bar{m}}(t)=1-\frac{\left[1-Q_{i}^{\bar{m}}(0)\right]\left[v_{j}+c_{j}^{m_{j}}(0)\right]}{v_{j}+c_{j}^{m_{j}}(t)}$ for all $t \leq t_{\bar{m}}^{\star}$,

2. $Q_{1}^{\bar{m}}(0) Q_{2}^{\bar{m}}(0)=0$,

3. $Q_{i}^{\bar{m}}\left(t_{\bar{m}}^{\star}\right)=1-z_{i}$ if $t_{\bar{m}}^{\star}$ satisfies $c_{i}^{m_{i}}\left(t_{\bar{m}}^{\star}\right)<w_{i}$,

4. $c_{i}^{m_{i}}\left(t_{\bar{m}}^{*}\right) \leq w_{i}$, and

5. $A_{i}^{\bar{m}}(t)=0$ for all $t<t_{\bar{m}}^{\star}$ and $\lim _{k \rightarrow \infty} A_{i}^{\bar{m}}(k)=1-z_{i}-Q_{i}^{\bar{m}}\left(t_{\bar{m}}^{\star}\right)$.

In equilibrium, if player $i$ believes that $j$ will never quit after time $t$ and $c_{i}^{m_{i}}(t)<w_{i}$, then flexible player $i$ will immediately quit at that time. There are two critical thresholds beyond which $j$ never quits; the time that player $j$ 's reputation reaches 1 and the time that $j$ 's escalation costs reaches his war cost $w_{j}$. Let $\kappa_{j}^{\bar{m}}$ satisfy $c_{j}\left(\kappa_{j}^{\bar{m}}\right)=w_{j}$ and $\tau_{j}^{\bar{m}}$ solve $Q_{j}^{\bar{m}}\left(\tau_{j}^{\bar{m}}\right)=1-z_{j}$. Thus, player $i$ never backs down after time $t_{j}^{\bar{m}}=\min \left\{\kappa_{j}^{\bar{m}}, \tau_{j}^{\bar{m}}\right\}$. Similar arguments hold for player $j$. If $t_{1}^{\bar{m}}<t_{2}^{\bar{m}}$ holds

6 Abreu and Sethi (2003) support the existence of commitment types from an evolutionary perspective and show that if players incur a cost of rationality, even if it is very small, the absence of such types is not compatible with evolutionary stability in a bargaining environment. 
and the dispute escalates until time $t_{1}^{\bar{m}}$, then flexible player 2 ends the game at this time for sure because he knows that player 1 will never quit beyond this point. As a result, the war of attrition game will end no later than time $t_{\bar{m}}^{\star}=\min \left\{t_{1}^{\bar{m}}, t_{2}^{\bar{m}}\right\}$. The next result formally proves these arguments.

Lemma 2: If a strategy profile $\sigma=\left\langle\mu_{i},\left\{Q_{i}^{\bar{m}}, A_{i}^{\bar{m}}\right\}_{\bar{m} \in M^{2}}\right\rangle_{i=1}^{2}$ constitutes a sequential equilibrium of the war of attrition game $G$, then for any $\bar{m} \in M^{2}$ and $i, j \in\{1,2\}$ with $i \neq j$,

1. flexible player $i$ never quits after time $t_{j}^{\bar{m}}=\min \left\{\kappa_{j}^{\bar{m}}, \tau_{j}^{\bar{m}}\right\}$, where

- $\kappa_{j}^{\bar{m}}$ solves $c_{j}^{m_{j}}\left(\kappa_{j}^{\bar{m}}\right)=w_{j}$, and

- $\tau_{j}^{\bar{m}}$ solves $c_{i}^{m_{i}}\left(\tau_{j}^{\bar{m}}\right)=\frac{v_{i}\left(1-z_{j}\right)+c_{i}^{m_{i}}(0)}{z_{j}}$, and

2. the game $G$ ends by time $t_{\bar{m}}^{\star}=\min \left\{t_{1}^{\bar{m}}, t_{2}^{\bar{m}}\right\}$.

Lemma 3: Suppose that a strategy profile $\sigma=\left\langle\mu_{i},\left\{Q_{i}^{\bar{m}}, A_{i}^{\bar{m}}\right\}_{\bar{m} \in M^{2}}\right\rangle_{i=1}^{2}$ constitutes a sequential equilibrium of the war of attrition game $G$. For any $\bar{m}=\left(m_{1}, m_{2}\right) \in M^{2}$, if $t_{i}^{\bar{m}}>t_{j}^{\bar{m}}$, then

$$
Q_{j}^{\bar{m}}(t)=1-\frac{v_{i}+c_{i}^{m_{i}}(0)}{v_{i}+c_{i}^{m_{i}}(t)} \quad \text { and } \quad Q_{i}^{\bar{m}}(t)=1-\frac{\left[1-Q_{i}^{\bar{m}}(0)\right]\left[v_{j}+c_{j}^{m_{j}}(0)\right]}{v_{j}+c_{j}^{m_{j}}(t)},
$$

where

$$
Q_{i}^{\bar{m}}(0)=1-\frac{z_{i}\left(v_{j}+c_{j}^{m_{j}}\left(t_{j}^{\bar{m}}\right)\right)}{v_{j}+c_{j}^{m_{j}}(0)}
$$

Definition 1: For any strategy profile $\sigma=\left\langle\mu_{i},\left\{Q_{i}^{\bar{m}}, A_{i}^{\bar{m}}\right\}_{\bar{m} \in M^{2}}\right\rangle_{i=1}^{2}$ that constitutes a sequential equilibrium of the war of attrition game $G$, player $i$ is called

(i) advantaged in the subgame following $\overline{\mathbf{m}}$ if $Q_{j}^{\bar{m}}(0)$ is strictly positive, and

(ii) advantaged in equilibrium $\sigma$ if he is advantaged on the equilibrium path, that is, he is advantaged in all the subgames following all $\bar{m}$ 's that are on the equilibrium path.

We know from Lemma 1 that in equilibrium, the equality $Q_{1}^{\bar{m}}(0) Q_{2}^{\bar{m}}(0)=0$ must hold for any $\bar{m} \in M^{2}$. Therefore, if $t_{i}^{\bar{m}}>t_{j}^{\bar{m}}$ holds, then player $i$ (and only player $i$ ) quits with a positive probability at time 0 in the subgame following $\bar{m}$. Thus, at most one player can be advantaged in equilibrium. The following result immediately follows from the last arguments.

Corollary 1: In any sequential equilibrium of the war of attrition game $G$, player $i$ is advantaged in the subgame following $\bar{m}$ if and only if the parameters of the game satisfy $t_{j}^{\bar{m}}>t_{i}^{\bar{m}}$. 


\section{Type (Hawkish vs. Dovish) and Intensity of the Tone}

This section aims to understand when the players prefer to choose the hawkish or the dovish stance and in what extent. For a sharper presentation of the underlying dynamics, I will analyze various variations of the war of attrition game with additional structure on the parameters.

Suppose that a strategy profile $\sigma=\left\langle\mu_{i},\left\{Q_{i}^{\bar{m}}, A_{i}^{\bar{m}}\right\}_{\bar{m} \in M^{2}}\right\rangle_{i=1}^{2}$ constitutes a sequential equilibrium of the war of attrition game G. For any message profile $\bar{m}$, players' strategies entail quitting at any time before $t_{\bar{m}}^{\star}$. Therefore, player $i$ 's expected payoff of quitting at any time $t$ where $0<t \leq t_{\bar{m}}^{\star}$ has a fixed value, and thus player $i$ 's expected payoff in the subgame following the message realization $\bar{m}$ must be equal to this fixed value.

More formally, we see by eq. [1] and Lemma 1 that for any small $\epsilon>0$, flexible player $i$ 's expected payoff of quitting at any time $t \leq t_{\bar{m}}^{\star}$ is

$$
\begin{aligned}
U_{i}^{q}(t, \sigma, \bar{m})= & v_{i} Q_{j}^{\bar{m}}(\epsilon)-w_{i} A_{j}^{\bar{m}}(\epsilon)+\left[-c_{i}^{m_{i}}(\epsilon)\right]\left[1-Q_{j}^{\bar{m}}(\epsilon)-A_{j}^{\bar{m}}(\epsilon)\right] \\
& +\left[\frac{v_{i}}{2}-c_{i}^{m_{i}}(\epsilon)\right]\left[Q_{j}^{\bar{m}}(\epsilon)-Q_{j}^{\bar{m}}\left(\epsilon^{-}\right)\right] \\
= & v_{i} Q_{j}^{\bar{m}}(\epsilon)-c_{i}^{m_{i}}(\epsilon)\left[1-Q_{j}^{\bar{m}}(\epsilon)\right] .
\end{aligned}
$$

Taking $\epsilon \rightarrow 0$ thus yields

$$
U_{i}^{q}(t, \sigma, \bar{m})=v_{i} Q_{j}^{\bar{m}}(0)-c_{i}^{m_{i}}(0)\left[1-Q_{j}^{\bar{m}}(0)\right]
$$

Similarly, we see by eq. [1] and Lemma 1 that player i's expected payoff of quitting at time $t_{\bar{m}}^{\star}$ is equal to

$$
U_{i}^{q}\left(t_{\bar{m}}^{\star}, \sigma, \bar{m}\right)=v_{i} Q_{j}^{\bar{m}}\left(t_{\bar{m}}^{\star}\right)-c_{i}^{m_{i}}\left(t_{\bar{m}}^{\star}\right)\left[1-Q_{j}^{\bar{m}}\left(t_{\bar{m}}^{\star}\right)\right],
$$

and one can easily check that it's value is equal to eq. [5]. Finally, eq. [2] and Lemma 1 imply that player $i$ 's expected payoff of attacking at any time $t \geq t_{\bar{m}}^{\star}$ is

$$
U_{i}^{a}\left(t_{\bar{m}}^{\star}, \sigma, \bar{m}\right)=v_{i} Q_{j}^{\bar{m}}\left(t_{\bar{m}}^{\star}\right)-w_{i}\left[1-Q_{j}^{\bar{m}}\left(t_{\bar{m}}^{\star}\right)\right] .
$$

By the fourth condition of Lemma 1, we have $c_{i}^{m_{i}}\left(t_{\bar{m}}^{\star}\right) \leq w_{i}$. That is, for any $t \leq t_{\bar{m}}^{\star}$, $U_{i}^{a}(t, \sigma, \bar{m}) \leq U_{i}^{q}(t, \sigma, \bar{m})$. Thus, flexible player $i$ 's expected payoff in the subgame following the message realization $\bar{m}$ is given by eq. [5].

For the rest of this section, I will consider linear escalation costs functions. In particular, for any message $m \in M$ and player $i$, I suppose that $c_{i}^{m}(t)=c_{i}^{m} t$ with $c_{i}^{m}>0$. Therefore, flexible player $i$ 's continuation payoff, following the subgame where message profile is $\bar{m}$, is simply 


$$
U_{i}^{\bar{m}}=v_{i} Q_{j}^{\bar{m}}(0)
$$

which is equal to $v_{i}\left[1-z_{j}\left(1+\frac{c_{i} t_{m}^{\star}}{v_{i}}\right)\right]$ when player $i$ is advantaged in the subgame following $\bar{m}$, or 0 otherwise. We know from Corollary 1 that player $i$ is advantaged in the subgame following $\bar{m}$ if and only if $t_{i}^{\bar{m}}<t_{j}^{\bar{m}}$. According to Lemma 2, for any $\bar{m}=\left(m_{1}, m_{2}\right) \in M^{2}$, the war of attrition game ends at time $t_{\bar{m}}^{\star}=$ $\min \left\{t_{1}^{\bar{m}}, t_{2}^{\bar{m}}\right\}$, where

$$
t_{1}^{\bar{m}}=\min \left\{\frac{w_{1}}{c_{1}^{m_{1}}}, \frac{v_{2}\left(1-z_{1}\right)}{z_{1} c_{2}^{m_{2}}}\right\}, \quad t_{2}^{\bar{m}}=\min \left\{\frac{w_{2}}{c_{2}^{m_{2}}}, \frac{v_{1}\left(1-z_{2}\right)}{z_{2} c_{1}^{m_{1}}}\right\} .
$$

\subsection{One-Sided Communication Under the Absence of War}

Let the game $G^{1}$ be the same as the game $G$ with only two differences: (1) in the first stage, only player 1 can choose his stance and (2) in the third stage, players cannot choose to attack. Furthermore, I suppose that $c_{1}^{d}<c_{2}<c_{1}^{h}$. Therefore, the game $G^{1}$ will end at time $t_{m}^{\star}=\min \left\{t_{1}^{m}, t_{2}^{m}\right\}$, where $t_{1}^{m}=\frac{v_{2}\left(1-z_{1}\right)}{z_{1} c_{2}}$ and $t_{2}^{m}=\frac{v_{1}\left(1-z_{2}\right)}{z_{2} c_{1}^{m}}$.

Moreover, player 1's expected payoff is

$$
U_{1}^{m}=v_{1}\left[1-z_{2}\left(1+\frac{v_{2}\left(1-z_{1}\right)}{v_{1} z_{1}} \frac{c_{1}^{m}}{c_{2}}\right)\right]
$$

if player 1 is advantaged in the subgame following $m$ (i. e., $t_{1}^{m}<t_{2}^{m}$ ). Note that $U_{1}^{m}$ is a decreasing function of $c_{1}^{m}$, and so player 1 can increase his game payoff by choosing a message that generates smaller escalation costs. Clearly, if player 1 can never be advantaged (i. e., $t_{2}^{m} \leq t_{1}^{m}$ for all $m \in M$ ), then player 1's equilibrium payoff will be 0 regardless of his message.

Proposition 1: If $c_{1}^{d}<\left(\frac{v_{1} z_{1}\left(1-z_{2}\right)}{v_{2} z_{2}\left(1-z_{1}\right)}\right) c_{2}$, then there exists a unique sequential equilibrium of the game $G^{1}$. In this equilibrium, player 1 is advantaged and chooses the dovish regime in the first stage.

If two players are similar in the sense that $v_{i}=v$ and $z_{i}=z$ for $i=1,2$, then the above inequality would become $c_{1}^{d}<c_{2}$, which is automatically satisfied by our assumption. Therefore, we could conclude that if two players are playing the war of attrition game under the absence of war and if only player 1 can choose his regime, then player 1 prefers to choose the dovish regime such that he keeps his escalation costs minimal. 


\subsection{One-Sided Communication Under the Shadow of War}

Let $G^{2}$ be the same as the game $G$ with only one difference: in the first stage, only player 1 can choose his regime. Similar to the previous section, I will continue to assume that $c_{1}^{d}<c_{2}<c_{1}^{h}$. Furthermore, let $v_{i}=v, w_{i}=w$, and $z_{i}=z$ hold for each $i$. According to Lemma 2, the equilibrium horizon of the game is a function of the players' war cost $w$. In particular, for each $m \in M$, the game $G^{2}$ will end at time $t_{m}^{\star}=\min \left\{t_{1}^{m}, t_{2}^{m}\right\}$, where $t_{1}^{m}=\min \left\{\frac{w}{c_{1}^{m}}, \frac{v(1-z)}{z c_{2}}\right\}$ and $t_{2}^{m}=\min \left\{\frac{w}{c_{2}}, \frac{v(1-z)}{z c_{1}^{m}}\right\}$. As a result, in equilibrium, player 1 should choose the hawkish regime when the benefit-cost ratio of the game is higher than the relative likelihood of a player being the resolved type. The next result formally proves this point.

Proposition 2: If $\frac{z}{1-z}<\frac{v}{w}$, then there exists a unique sequential equilibrium of the game $G^{2}$, in which player 1 is advantaged and choosing the hawkish regime in the first stage. However, if the reverse of this inequality holds, then there is a unique sequential equilibrium of the game $G^{2}$, in which player 1 is advantaged but choosing the dovish regime in the first stage.

If only player 1 has had the option of attacking, and so player 2 can never attack, then there would exist a unique equilibrium where player 1 was advantaged and choosing the dovish regime when $\frac{v}{w}<\frac{z}{1-z}$. However, there would exist a continuum of equilibrium - in all of which player 1 is advantaged - when $\frac{z}{1-z}<\frac{v}{w}$ because player 1 would be indifferent between the dovish and the hawkish regimes.

These two subsections show that player 1, who is the only player that can choose his escalation costs in the game, is the advantaged player in equilibrium, and this conclusion is correct whether or not players have the option to attack. However, the advantaged player's regime varies depending on the benefit-cost ratio of the dispute and the relative likelihood of a player being the resolved type.

\subsection{Two-Sided Communication}

In this section, I consider the war of attrition game $G$, where both players can choose a regime. For sharper results, I will suppose that $c_{2}^{d}<c_{1}^{d}<c_{2}^{h}<c_{1}^{h}, v_{i}=v$, $w_{i}=w$, and $z_{i}=z$ for each $i$.

Proposition 3: If $\frac{z}{1-z}<\frac{v}{w}$, then in any sequential equilibrium of the game $G$, player 1 1. is advantaged and choosing the hawkish regime in the first stage, and

2. attacks (at some time after $\frac{w}{c_{1}^{h}}$ ) with probability $\frac{v}{v+w}-z$. 
However, if $\frac{v}{w}<\frac{z}{1-z}$, then in any sequential equilibrium of the game $G$, player 2 is advantaged and choosing the dovish regime in the first stage.

\subsection{Continuous Message Space and Intensity}

Similar to the previous section, I suppose that both players can choose escalation costs coefficients $\left(c_{i}\right)$, and $v_{i}=v, w_{i}=w$, and $z_{i}=z$ hold for each $i$. Furthermore, I suppose that message space is continuous, and so for $i=1,2$, $c_{i} \in\left[c^{\min }, c^{\max }\right]$ with $0<c^{\min }<c^{\max }<\infty$. The lower (or the upper) bound represents the most dovish (hawkish) tone a player can pick.

Proposition 4: Suppose that $c_{j} \in\left[c^{\min }, c^{\max }\right]$ for $j \in\{1,2\}$. Then the best response correspondence for the flexible type of player $i \in\{1,2\}$ with $i \neq j$ is

$$
B R_{i}\left(c_{j}\right)=\left\{\begin{array}{cl}
\left(c_{j}, c^{\max }\right], & \text { if } c_{j}<c^{\max } \text { and } \frac{z}{1+z}<\frac{v}{w}, \\
{\left[c^{\min }, c^{\max }\right]} & \text { if } c_{j}=c^{\max } \text { and } \frac{z}{1+z}<\frac{v}{w}, \\
\left\{c^{\min }\right\}, & \text { if } c^{\min }<c_{j} \text { and } \frac{z}{1+z}>\frac{v}{w} \\
{\left[c^{\min }, c^{\max }\right]} & \text { if } c^{\min }=c_{j} \text { and } \frac{z}{1+z}>\frac{v}{w} .
\end{array}\right.
$$

Therefore, more dovish tone increases a player's payoff as long as the dovish regime is optimal and the opponent has not chosen the most dovish regime $c^{\mathrm{min}}$. On the other hand, a more hawkish tone never benefits a player if it is the optimal regime for the player. Nevertheless, in all sequential equilibrium of the war of attrition game $G$, at least one of the players will certainly choose the most hawkish regime $c^{\max }$ whenever $\frac{z}{1+z}<\frac{v}{w}$.

\section{Concluding Remarks}

This paper examines a two-player war of attrition game in continuous-time. A player can build his reputation by mimicking the resolved type and escalating the dispute. Higher reputation for resolve intimidates the rival and forces him to play a mixed strategy in which he quits with a greater probability. By choosing his regime at the beginning of the game, players can control how fast they will build their reputation. The dovish regime gives the ability of building reputation at a faster rate. The threat of a costly war introduces some sort of deadline effect for reputation building. A flexible player does not quit beyond a time at which his escalation costs exceed his war cost. Therefore, by choosing the hawkish regime, players commit to attack with a positive probability after this time, and so to a shorter time horizon for reputation building. 
Our results show that choosing the hawkish regime (and so imposing a shorter deadline) is optimal when the benefit-cost ratio of the dispute is higher than the relative likelihood of a player being the resolved type. Otherwise, choosing the dovish regime (i. e., building reputation at a faster rate) is the optimal action. These results were speculated in Ozyurt (2014), but formally proved in the current paper. In addition, the current paper shows that having more hawkish tone never benefits a player if hawkish regime is already the optimal one. However, choosing more dovish tone increases a player's payoff when the dovish regime is optimal. Furthermore, hawkish tone is not advantageous in a dispute if the players do not have the option of finishing the dispute with a costly war. Although a linear functional form for the escalation costs is an important restriction, it does not alter the qualitative nature of our results.

Funding: This research was supported by the Marie Curie International Reintegration Grant (\# 256486) within the European Community Framework Programme.

\section{Appendix}

Proof of Lemma 1: Let $\sigma=\left\langle\mu_{i},\left\{Q_{i}^{\bar{m}}, A_{i}^{\bar{m}}\right\}_{\bar{m} \in M^{2}}\right\rangle_{i=1}^{2}$ be a sequential equilibrium of the game $G$. For any $\bar{m} \in M^{2}$ and $i \in\{1,2\}$, let $\kappa_{i}^{\bar{m}}=\inf \left\{t \geq 0 \mid Q_{i}^{\bar{m}}(t)=\right.$ $\left.\lim _{k \rightarrow \infty} Q_{i}^{\bar{m}}(k)\right\}$. The optimality of equilibrium implies that player $i$ does not quit beyond time $t$, satisfying $c_{i}(t)=w_{i}$. Therefore, $\kappa_{i}^{\bar{m}}$ is finite. The proofs of the following arguments $(i)-(v)$ directly follow from the proof of Proposition 1 in Ozyurt (2014). Therefore, I skip the details.

(i) $\kappa_{1}^{\bar{m}}=\kappa_{2}^{\bar{m}}$ : A flexible player does not delay quitting once he knows that his opponent will never quit.

(ii) If $Q_{i}^{\bar{m}}$ jumps at $t$, then $Q_{j}^{\bar{m}}$ is constant at some $\epsilon$-neighborhood of $t$.

(iii) If $Q_{i}^{\bar{m}}$ is constant between $\left(t^{\prime}, t^{\prime \prime}\right)$, then so is $Q_{j}^{\bar{m}}$.

(iv) There is no interval $\left(t^{\prime}, t^{\prime \prime}\right)$ with $t^{\prime \prime}<\kappa_{1}^{\bar{m}}$ on which $Q_{1}^{\bar{m}}$ and $Q_{2}^{\bar{m}}$ are constant.

(v) If $t^{\prime}<t^{\prime \prime}<\kappa_{1}^{\bar{m}}$, then $Q_{i}^{\bar{m}}\left(t^{\prime}\right)<Q_{i}^{\bar{m}}\left(t^{\prime \prime}\right)$ for $i=1,2$.

From $(i)-(v)$, it follows that $Q_{1}^{\bar{m}}$ and $Q_{2}^{\bar{m}}$ must be continuous and strictly increasing on $\left[0, t_{\bar{m}}^{\star}\right]$, where $t_{\bar{m}}^{\star}=\kappa_{1}^{\bar{m}}=\kappa_{2}^{\bar{m}}$. That is, flexible players are indifferent between quitting at time $t<t_{\bar{m}}^{*}$ and waiting for an infinitesimal period $\Delta$ and then quit at time $t+\Delta<t_{\bar{m}}^{*}$. But then, player $i$ must be quitting with a constant hazard rate $\lambda_{i}=\frac{c_{j}^{\prime}(t)}{v_{j}+c_{j}(t)}$, and thus $Q_{i}^{\bar{m}}(t)=1-\frac{\left[1-Q_{i}^{\bar{m}}(0)\right]\left[v_{j}+c_{j}^{m_{j}}(0)\right]}{v_{j}+c_{j}^{m_{j}}(t)}$ for all $t \leq t^{\star}$. By (ii), both $Q_{1}^{\bar{m}}(0)$ and $Q_{2}^{\bar{m}}(0)$ cannot be positive, implying that $Q_{1}^{\bar{m}}(0) Q_{2}^{\bar{m}}(0)=0$. 
Because $Q_{i}^{\bar{m}}$ is strictly increasing on $\left[0, t_{\bar{m}}^{\star}\right]$, it must be the case that $c_{i}\left(t_{\bar{m}}^{\star}\right) \leq w_{i}$ for each $i$. Therefore, $A_{i}^{\bar{m}}(t)=0$ for all $t<t_{\bar{m}}^{\star}$. Since escalating forever is costlier than attacking and $Q_{i}^{\bar{m}}(t)+A_{i}^{\bar{m}}(t) \leq 1-z_{i}$ for all $t \geq 0$, we must have $\lim _{k \rightarrow \infty} A_{i}^{\bar{m}}(k)=1-z_{i}-Q_{i}^{\bar{m}}\left(t_{\bar{m}}^{\star}\right)$ for $i=1$, 2. Finally, probability that player $i$ quits by time $t_{\bar{m}}^{\star}$ conditional on the event that he chooses $m_{j}$ is less than (or equal to) 1, implying that $Q_{i}^{\bar{m}}\left(t^{\star}\right) \leq 1-z_{i}$.

Finally, since a flexible player will not delay quitting once he knows his opponent will never quit, and will not attack before time $t_{\bar{m}}^{\star}$, we can conclude that $Q_{i}^{\bar{m}}\left(t^{\star}\right)=1-z_{i}$ for $i=1,2$ if $t_{\bar{m}}^{\star}$ satisfies $c_{i}^{m_{i}}\left(t_{\bar{m}}^{\star}\right)<w_{i}$. However, when $c_{i}^{m_{i}}\left(t_{\bar{m}}^{\star}\right)=w_{i}$, player $i$ will be indifferent between quitting at time $t_{\bar{m}}^{\star}$ and attacking at (or after) time $t_{\bar{m}}^{\star}$. Therefore, we must have $Q_{i}^{\bar{m}}\left(t^{\star}\right)<1-z_{i}$ if $c_{i}^{m_{i}}\left(t_{\bar{m}}^{\star}\right)=w_{i}$.

Proof of Lemma 2: The discussions that follow Lemma 1 prove the first part (i.e., 1), so I will not repeat them here. Regarding the equality in $\mathbf{1}$ - ii, note that $\tau_{j}^{\bar{m}}$ solves $Q_{j}^{\bar{m}}\left(\tau_{j}^{\bar{m}}\right)=1-z_{j}$ under the assumption $Q_{j}^{\bar{m}}(0)=0$. Moreover, those discussions already prove that the game will end no later than $t_{\bar{m}}^{\star}$. I will complete the proof by showing that the game $G$ will continue until time $t_{\bar{m}}^{\star}$ with a positive probability. Suppose that the game ends prior to time $\hat{t}$ with certainty, where $\hat{t}<t_{\bar{m}}^{\star}$. According to the equilibrium strategies given by Lemma $1, Q_{i}^{\bar{m}}(0)>0$ must hold for $i=1,2$ because otherwise the condition $Q_{i}^{\bar{m}}(\hat{t})=1-z_{i}$ will not hold for each $i$ (note that if $\hat{t}<t_{i}^{\bar{m}}$, then $\left.c_{i}(\hat{t})<w_{i}\right)$. However, having $Q_{1}^{\bar{m}}(0)>0$ and $Q_{2}^{\bar{m}}(0)>0$ simultaneously contradicts the optimality of the equilibrium (recall the condition 2 of Lemma 1). Hence, in equilibrium, given that flexible players randomize the timing of quitting, escalation continues until time $t_{\bar{m}}^{\star}$ with some positive probability and stops at this time with certainty.

Proof of Lemma 3: Since $t_{i}^{\bar{m}}>t_{j}^{\bar{m}}$, we have $t_{\bar{m}}^{\star}=t_{j}^{\bar{m}}$. Therefore, $\tau_{i}^{\bar{m}}, \kappa_{i}^{\bar{m}}>t_{\bar{m}}^{\star}$. Recall that $\tau_{i}^{\bar{m}}$ is the time satisfying $Q_{i}^{\bar{m}}\left(\tau_{i}^{\bar{m}}\right)=1-z_{i}$ if $Q_{i}^{\bar{m}}(0)=0$. Since the game ends before time $\tau_{i}^{\bar{m}}$ and $\kappa_{i}^{\bar{m}}>t_{\bar{m}}^{\star}$ holds, we must have $Q_{i}^{\bar{m}}\left(t_{\bar{m}}^{\star}\right)=1-z_{i}$. Hence, we have $Q_{i}^{\bar{m}}(0)>0$. According to the second condition of Lemma 1, we should also have $Q_{1}^{\bar{m}}(0) Q_{2}^{\bar{m}}(0)=0$. The last condition with $Q_{i}^{\bar{m}}(0)>0$ implies that $Q_{j}^{\bar{m}}(0)=0$. However, $Q_{j}^{\bar{m}}(0)=0$ implies $Q_{j}^{\bar{m}}(t)=1-\frac{v_{i}+c_{i}^{m_{i}}(0)}{v_{i}+c_{i}^{m_{i}}(t)}$. Moreover, since $Q_{i}^{\bar{m}}\left(t_{j}^{\bar{m}}\right)=$ $1-\frac{\left[1-Q_{i}^{\bar{m}}(0)\right]\left[v_{j}+c_{j}^{m_{j}}(0)\right]}{v_{j}+c_{j}^{m_{j}}\left(t_{j}^{\bar{m}}\right)}=1-z_{i}$, we have $Q_{i}(0)=1-\frac{z_{i}\left(v_{j}+c_{j}^{m_{j}}\left(t_{j}^{\bar{m}}\right)\right)}{v_{j}+c_{j}^{m_{j}}(0)}$. This completes the proof.

Proof of Proposition 1: Consider the following strategies: In the first stage, player 1 chooses the dovish regime with probability 1 (i. e., $\mu_{1}(d)=1$ ). In the second stage, following the dovish regime, players' strategies are given by Lemma 3, that is, $Q_{1}^{d}(t)=1-\frac{v_{2}}{v_{2}+c_{2} t}$ and $Q_{2}^{d}(t)=1-\frac{\left[1-Q_{2}^{d}(0)\right] v_{1}}{v_{1}+c_{1}^{d} t}$ for all $t \leq t_{d}^{\star}=\frac{v_{2}\left(1-z_{1}\right)}{z_{1} c_{2}}$, 
where $Q_{2}^{d}(0)=1-\frac{z_{2}\left(v_{1}+c_{1}^{d} t_{d}^{\star}\right)}{v_{1}}$. Similarly, Lemma 3 characterizes the players' equilibrium strategies for the third stage when player 1 chooses the hawkish regime in the first period.

Given $Q_{j}^{m}$, flexible player $i$ is indifferent between quitting at time $t^{\prime}$ and waiting for some time and then quitting at time $t^{\prime \prime}$, where $0 \leq t^{\prime}<t^{\prime \prime} \leq t_{m}^{\star}$, where $m \in M$. Hence, any mixed strategy on the support $\left[0, t_{m}^{\star}\right]$, in particular, $Q_{i}^{m}$ is optimal for player $i$. Finally, because player 1's payoff decreases with $c_{1}^{m}$, $\mu_{1}(d)=1$ is also optimal. Hence, these strategies indeed form equilibrium. Uniqueness of the equilibrium is implied by the fact that the payoff of player 1 is strictly higher under dovish regime (see eq. [6]) and by the third stage equilibrium strategies that are characterized in Section 3.

Proof of Proposition 2: Similar to the strategies given in the proof of Proposition 1, Lemma 1-3 characterize the equilibrium strategies of the third stage of the game $G^{2}$. In order to find player 1's first stage equilibrium strategy, consider first the case where player 1 chooses the dovish regime. In this case, $t_{1}^{d}=\min \left\{\frac{w}{c_{1}^{d}}, \frac{v(1-z)}{c_{2} z}\right\}$ and $t_{2}^{d}=\min \left\{\frac{w}{c_{2}}, \frac{v(1-z)}{c_{1}^{d} z}\right\}$. First note that $\frac{w}{c_{2}}<\frac{w}{c_{1}^{d}}$ and $\frac{v(1-z)}{c_{2} z}<\frac{v(1-z)}{c_{1}^{d} z}$. Therefore, according to Corollary 1 , if $\frac{z}{1-z}<\frac{v}{w}$, then player 2 is advantaged. If the inequality is reversed, then player 1 is advantaged.

On the other hand, if player 1 chooses the hawkish regime, then $t_{1}^{h}=$ $\min \left\{\frac{w}{c_{1}^{h}}, \frac{v(1-z)}{c_{2} z}\right\}$ and $t_{2}^{h}=\min \left\{\frac{w}{c_{2}}, \frac{v(1-z)}{c_{1}^{h} z}\right\}$. Since $\frac{w}{c_{1}^{h}}<\frac{w}{c_{2}}$ and $\frac{v(1-z)}{c_{1}^{h} z}<\frac{v(1-z)}{c_{2} z}$, Corollary 1 implies that player 1 is advantaged if and only if $\frac{w}{c_{1}^{h}}<\frac{v(1-z)}{c_{1}^{h} z}$, equivalently if and only if $\frac{z}{1-z}<\frac{v}{w}$.

Thus, if $\frac{z}{1-z}<\frac{v}{w}$, then player 1's optimal strategy is to choose the hawkish regime, and if $\frac{v}{w}<\frac{z}{1-z}$, then player 1's optimal strategy is to choose the dovish regime. Finally, the uniqueness is implied by player 1's first stage strategies and by the the third stage equilibrium strategies that are characterized in Section 3.

Proof of Proposition 3: The equilibrium strategies in the third stage of the game are already characterized in Section 3. Recall that for any $\bar{m}=\left(m_{1}, m_{2}\right) \in M^{2}$, the war of attrition game ends at time $t_{\bar{m}}^{\star}=\min \left\{t_{1}^{\bar{m}}, t_{2}^{\bar{m}}\right\}$, where $t_{1}^{\bar{m}}=\min \left\{\frac{w_{1}}{c_{1}^{m_{1}}}, \frac{v_{2}\left(1-z_{1}\right)}{z_{1} c_{2}^{m_{2}}}\right\}$ and $t_{2}^{\bar{m}}=\min \left\{\frac{w_{2}}{c_{2}^{m_{2}}}, \frac{v_{1}\left(1-z_{2}\right)}{z_{2} c_{1}^{m_{1}}}\right\}$. In order to characterize the first stage equilibrium strategies, we need to consider four cases. 
Case 1: Both players choose the dovish regime: Since $\frac{w}{c_{1}^{d}}<\frac{w}{c_{2}^{d}}$ and $\frac{v(1-z)}{c_{1}^{d} z}<\frac{v(1-z)}{c_{2}^{d} z}$, we have

- $\quad t_{d d}^{\star}=\frac{w}{c_{1}^{d}}$ (i. e., player 1 is advantaged) whenever $\frac{w}{c_{1}^{d}}<\frac{v(1-z)}{c_{1}^{d} z}$, which is equivalent to $\frac{z}{1-z}<\frac{v}{w}$, and

$-\quad t_{d d}^{\star}=\frac{v(1-z)}{c_{1}^{d} z}$ (i. e., player 2 is advantaged) whenever $\frac{v(1-z)}{c_{1}^{d} z}<\frac{w}{c_{1}^{d}}$ (i. e., $\frac{v}{w}<\frac{z}{1-z}$ ).

Case 2: Player 1 and 2 choose the hawkish and the dovish regime, respectively: Since $\frac{w}{c_{1}^{h}}<\frac{w}{c_{2}^{d}}$ and $\frac{v(1-z)}{c_{1}^{h} z}<\frac{v(1-z)}{c_{2}^{d} z}$, we have

- $\quad t_{h d}^{\star}=\frac{w}{c_{1}^{h}}$ (i. e., player 1 is advantaged) whenever $\frac{w}{c_{1}^{h}}<\frac{v(1-z)}{c_{1}^{h} z}$ (i. e., $\frac{z}{1-z}<\frac{v}{w}$ ), and - $\quad t_{h d}^{\star}=\frac{v(1-z)}{c_{1}^{h} z}$ (i. e., player 2 is advantaged) whenever $\frac{v(1-z)}{c_{1}^{h} z}<\frac{w}{c_{1}^{h}}$ (i. e., $\frac{v}{w}<\frac{z}{1-z}$ ).

Case 3: Player 1 and 2 choose the dovish and the hawkish regime, respectively: Since $\frac{w}{c_{2}^{h}}<\frac{w}{c_{1}^{d}}$ and $\frac{v(1-z)}{c_{2}^{h} z}<\frac{v(1-z)}{c_{1}^{d} z}$, we have

- $\quad t_{d h}^{\star}=\frac{w}{c_{2}^{h}}$ (i. e., player 2 is advantaged) whenever $\frac{w}{c_{2}^{h}}<\frac{v(1-z)}{c_{2}^{h} z}$ (i. e., $\frac{z}{1-z}<\frac{v}{w}$ ), and

$-\quad t_{d h}^{\star}=\frac{v(1-z)}{c_{2}^{h} z}$ (i. e., player 1 is advantaged) whenever $\frac{v(1-z)}{c_{2}^{h} z}<\frac{w}{c_{2}^{h}}$ (i. e., $\frac{v}{w}<\frac{z}{1-z}$ ).

Case 4: Both players choose the hawkish regime: Since $\frac{w}{c_{1}^{h}}<\frac{w}{c_{2}^{h}}$ and $\frac{v(1-z)}{c_{1}^{h} z}<\frac{v(1-z)}{c_{2}^{h} z}$, we have

$-\quad t_{h h}^{\star}=\frac{w}{c_{1}^{h}}$ (i. e., player 1 is advantaged) whenever $\frac{w}{c_{1}^{h}}<\frac{v(1-z)}{c_{1}^{h} z}$ (i. e., $\frac{z}{1-z}<\frac{v}{w}$ ), and

- $\quad t_{h h}^{\star}=\frac{v(1-z)}{c_{1}^{h} z}$ (i. e., player 2 is advantaged) whenever $\frac{v(1-z)}{c_{1}^{h} z}<\frac{w}{c_{1}^{h}}$ (i. e., $\frac{v}{w}<\frac{z}{1-z}$ ).

First suppose that $\frac{z}{1-z}<\frac{v}{w}$. When player 1 chooses the dovish regime, player 2's best response is to choose hawkish regime (by cases 1 and 3). However, when player 2 takes the hawkish regime, player 1's best response is to choose the hawkish regime as well (by cases 3 and 4). Hence, if benefit-cost ratio of the dispute exceeds the relative likelihood of a player being the resolved type, then there exists no sequential equilibrium in which player 1 takes a dovish regime. However, when player 1 takes a hawkish regime, player 2 is indifferent between the hawkish and dovish messages simply because player 1 is advantaged in either case (see cases 2 and 4). Because hawkish regime is a best response for player 1 and the game ends at time $w / c_{1}^{h}$ regardless of player 2's regime, we can conclude that there is a continuum of equilibrium, in which player 1 is advantaged and choosing the hawkish regime, and the horizon of the game is $w / c_{1}^{h}$. By Lemma 1 , the probability of attacking is $\lim _{k \rightarrow \infty} A_{1}^{h h}(k)=$ $1-z-Q_{1}^{h h}\left(w / c_{1}^{h}\right)=\frac{v}{v+w}-z$.

Now suppose that $\frac{v}{w}<\frac{z}{1-z}$. When player 2 chooses the hawkish message, player 1's best response is to choose the dovish message (by cases 3 and 4). 
However, when 1 chooses the dovish regime, player 2's best response is to choose the dovish regime, not the hawkish regime (by cases 1 and 3). Hence, if the relative likelihood of a player being the resolved type exceeds the benefitcost ratio of the dispute, then there exists no sequential equilibrium in which player 2 takes a hawkish regime. However, when player 2 chooses the dovish regime, both dovish and hawkish messages are best response for player 1 (by cases 1 and 2) because player 2 is advantaged in each case, and thus, player 1's payoff is 0 . Because the dovish regime is a best response for player 2 regardless of player 1's regime, we can conclude that there is a continuum of equilibrium, in which player 2 is advantaged and choosing the dovish regime. In these equilibria, the game ends at times $\frac{c(1-z)}{c_{1}^{d} z}$ and $\frac{c(1-z)}{c_{1}^{h} z}$, when player 1's realized message is $d$ and $h$, respectively. Therefore, in all equilibria, both players' reputations simultaneously converge to one at the end of the game, so that no player attacks with a positive probability.

Proof of Proposition 4: Suppose that $c^{\min }<c_{j}<c^{\max }$. Proposition 3 implies that if $\frac{z}{1+z}<\frac{v}{w}$, then player $i$ can be advantaged by choosing a hawkish tone $c_{i}$ such that $c_{j}<c_{i}$. In that case, the horizon of the game $\left(t^{\star}\right)$ is equal to $\frac{w}{c_{i}}$ (see the proof of Proposition 3). By substituting this value in player i's payoff function $U_{i}=v\left[1-z\left(1+\frac{c_{i} t^{*}}{v}\right)\right]$ we get $U_{i}=v\left[1-z\left(1+\frac{w}{v}\right)\right]$, which is independent of $c_{i}$. Thus, player $i$ 's expected payoff does not chance with $c_{i}$ as long as $c_{j}<c_{i}$.

On the other hand, if $\frac{z}{1+z}>\frac{v}{w}$, then player $i$ can be advantaged by choosing a dovish regime $c_{i}$ such that $c_{i}<c_{j}$. In this case, the horizon of the game $\left(t^{\star}\right)$ is equal to $\frac{v(1-z)}{c_{j} z}$ (see the proof of Proposition 3). By substituting this value in player $i$ 's payoff function we get $U_{i}=v\left[1-z\left(1+\frac{c_{i}(1-z)}{c_{j} z}\right)\right]$, which decreases with $c_{i}$. Thus, player $i$ 's best response to $c_{j}$ is picking the most dovish tone that is less than $c_{j}$, which is $c^{\mathrm{min}}$. Verifying the cases where $c_{j}$ takes the boundary values is very similar, and so omitted.

\section{References}

Abreu, D., and F. Gul. 2000. "Bargaining and Reputation." Econometrica 68 (1):85-117. Abreu, D., and R. Sethi. 2003. "Evolutionary Stability in a Reputational Model of Bargaining." Games and Economic Behavior 44 (2):195-216.

Atakan, A. E., and M. Ekmekci. 2014. "Bargaining and Reputation in Search Markets." The Review of Economic Studies 81 (1):1-29. 
Bishop, D. T., C. Cannings, and J. Maynard Smith. 1978. "The War of Attrition with Random Rewards." Journal of Theoretical Biology 74 (3):377-88.

Bliss, C., and B. Nalebuff. 1984. "Dragon-Slaying and Ballroom Dancing: The Private Supply of a Public Good.” Journal of Public Economics 25 (1):1-12.

Bulow, J., and P. Klemperer. 1999. "The Generalized War of Attrition.” American Economic Review 89 (1):175-89.

Chatterjee, K., and L. Samuelson. 1987. "Bargaining with Two-Sided Incomplete Information: An Infinite Horizon Model with Alternating Offers." The Review of Economic Studies 54 (2):175-92.

Compte, O., and P. Jehiel. 2002. "On the Role of Outside Options in Bargaining with Obstinate Parties." Econometrica 70 (4):1477-517.

Fearon, J. D. 1994. "Domestic Political Audiences and the Escalation of International Disputes." American Political Science Review 88 (3):577-92.

Fudenberg, D., R. Gilbert, J. Stiglitz, and J. Tirole. 1983. "Preemption, Leapfrogging and Competition in Patent Races." European Economic Review 22 (1):3-31.

Fudenberg, D., and J. Tirole. 1986. "A Theory of Exit in Duopoly." Econometrica $54(4): 943-60$.

Ghemawat, P., and B. Nalebuff. 1985. “Exit.” The RAND Journal of Economics 16 (2):184-94.

Guisinger, A., and A. Smith. 2002. "Honest Threats the Interaction of Reputation and Political Institutions in International Crises." Journal of Conflict Resolution 46 (2):175-200.

Hendricks, K., A. Weiss, and C. Wilson. 1988. "The War of Attrition in Continuous Time with Complete Information.” International Economic Review 29 (4):663-80.

Kambe, S. 1999. "Bargaining with Imperfect Commitment." Games and Economic Behavior 28 (2):217-37.

Kreps, D. M., and R. Wilson. 1982. "Reputation and Imperfect Information." Journal of Economic Theory 27 (2):253-79.

Maynard Smith, J. 1974. "The Theory of Games and the Evolution of Animal Conflicts." Journal of Theoretical Biology 47 (1):209-21.

Milgrom, P., and J. Roberts. 1982. "Predation, Reputation, and Entry Deterrence." Journal of Economic Theory 27 (2):280-312.

Milgrom, P. R., and R. J. Weber. 1985. "Distributional Strategies for Games with Incomplete Information." Mathematics of Operations Research 10 (4):619-32.

Myerson, R. B. 1991. Game Theory: Analysis of Conflict. Cambridge, Massachusetts: Harvard University Press.

Nalebuff, B., and J. Riley. 1985. "Asymmetric Equilibria in the War of Attrition." Journal of Theoretical Biology 113 (3):517-27.

Ordover, J. A., and A. Rubinstein. 1986. "A Sequential Concession Game with Asymmetric Information." The Quarterly Journal of Economics 101 (4):879-88.

Osborne, M. J. 1985. “The Role of Risk Aversion in a Simple Bargaining Model.” Game Theoretic Models of Bargaining, 181-213.

Ozyurt, S. 2014. "Audience Costs and Reputation in Crisis Bargaining." Games and Economic Behavior 88:250-9.

Ozyurt, S. 2015. "Searching for a Bargain: Power of Strategic Commitment." American Economic Journal: Microeconomics 7 (1):320-53.

Ponsati, C., and J. Sakovics. 1995. "The War of Attrition with Incomplete Information." Mathematical Social Sciences 29 (3):239-54. 
Riley, J. G. 1980. "Strong Evolutionary Equilibrium and the War of Attrition." Journal of Theoretical Biology 82 (3):383-400.

Sartori, A. E. 2002. "The Might of the Pen: A Reputational Theory of Communication in International Disputes." International Organization 56 (1):121-49.

Smith, A. 1998. "International Crises and Domestic Politics." American Political Science Review $92(3): 623-38$.

Snyder, J., and E. D. Borghard. 2011. "The Cost of Empty Threats: A Penny, Not a Pound.” American Political Science Review 105:437-56.

Trachtenberg, M. 2012. “Audience Costs: An Historical Analysis.” Security Studies 21:3-42. 